\title{
A seminal monograph: Mackay and Burnet's Autoimmune diseases
}

T oday, more than 80 specific autoimmune diseases are recognised, likely affecting over $5 \%$ of the Australian population. ${ }^{1}$ In terms of the economic burden they place on society, autoimmune diseases rank closely behind cancer, cardiovascular disease and respiratory disease. ${ }^{2}$ Fifty years ago, however, things were rather different. The concept of autoimmunity was still very much in its infancy, with many highly regarded clinicians and scientists dismissing the proposition out of hand. ${ }^{3}$

The seminal monograph Autoimmune diseases: pathogenesis, chemistry and therapy, written in 1961 by Australians Ian Reay Mackay and Sir Frank Macfarlane Burnet, ${ }^{4}$ has had an important influence on the recognition, acceptance and understanding of autoimmune disorders and was the first publication solely on the topic of autoimmunity. It was commissioned by American publisher Charles Thomas and first published in late 1962. Spanish and Japanese translations appeared in 1965 and 1967, respectively. The monograph was aimed at practising clinicians rather than at bench scientists and was written shortly after the publication of Burnet's classic monograph The clonal selection theory of acquired immunity, ${ }^{5}$ which also provided a sound theoretical background for the existence of autoimmunity.

Sir Macfarlane Burnet, OM, AK, KBE, the senior author, was arguably one of the greatest medical scientists of the 20th century. He was born in Victoria in 1899, graduated MB BS from the University of Melbourne, and served as Director of the Walter and Eliza Hall Institute (WEHI) from 1944 to 1965. WEHI, established in 1915, is one of Australia's leading research facilities, particularly in the fields of immunology, cancer and molecular biology. In 1960, Burnet was a co-recipient of the Nobel Prize in Physiology or Medicine. He died in 1985. The first author, Ian Mackay, AM, was born in Victoria in 1922 and also graduated MB BS from the University of Melbourne. He became head of the Clinical Research Unit of WEHI in 1963 and initiated a comprehensive study into autoimmunity.

In their 1962 monograph, Mackay and Burnet were able to define autoimmune disease "as a condition in which structural or functional damage is produced by the action of immunologically competent cells or antibodies against normal components of the body" and which arose by "the emergence of forbidden clones" of lymphocytes. ${ }^{4}$ The first three chapters were written by Burnet and provided PATHOOENESIS, CHEMTSTRY
and THERAPY

AUTOIMMUNE DISEASES

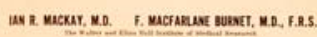

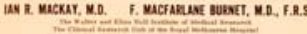

Macfarlane Burnethas achieved international asight into immunological problems earned him the Nobel Prize in Medicine in 1960.
hocher Doctor Mackay's work on the immunological aspects of chronic liver disease and sy stemic
lupus erythematosus has also gained world lupus erythematosus has also gained world
acclaim. The present publication is the fruit of their joint investigationg carefally inter: woven with researchen of other leaders in this field- - a vivid deseription of diseases immune processes in man.

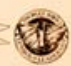

Historical facts

- Mackay and Burnet's Autoimmune diseases, published in 1962, marked the beginning of autoimmunity as a clinical science and led to the future acceptance of the existence of autoimmunity.

- While there is still controversy regarding the mechanisms of autoimmunity, the authors' insightful hypothesis based on clonal selection theory and the emergence of "forbidden clones", due to somatic mutations, is still current, with recent evidence giving further credence to this hypothesis.

- We salute Mackay and Burnet on the 50th anniversary of this seminal publication. It is particularly pleasing that it has an iconic Australian origin.

definitions of immunological terms (a new language at that time), the theoretical background for normal immune responsiveness through clonal selection, and the mechanism for loss of immunological tolerance through the emergence of "forbidden" (autoreactive) clones. The next eight chapters were written by Mackay and detailed diseases that, in his opinion, were likely to be autoimmune. These diseases all had characteristic clinical or pathological markers of autoimmunity, including the presence of an autoantibody, hypergammaglobulinaemia, tissue deposition of immune complexes, lymphocytic and plasma cell accumulation in affected tissues, therapeutic benefit from corticosteroids, and overlap of differing autoimmune manifestations in the same patient (page 16).

Mackay and Burnet observed that autoimmunity may manifest in single organs or in a diffuse pattern, frequently shows a female predominance, and has a fluctuating course. They noted that genetic factors are also commonly seen, and initiating or triggering processes (eg, sunburn, viral and bacterial infections, chronic sepsis, pregnancy or drugs) could be identified in some patients (pages 19 and 263). The final two chapters included an analysis of therapeutic options - corticosteroids, cytotoxic drugs, antimalarial and other drugs, ionising radiation and surgical excision of lymphoid tissue.

Following the monograph's publication, many reviews appeared in medical journals. Most praised the work, but not all - one in the Journal of the American Medical Association even questioned the timing, relevance and targeted audience for a book on "so-called 'autoimmune' diseases". ${ }^{6}$ However, with the benefit of hindsight, we can now reflect on Mackay and Burnet's insightful observations in this pioneering monograph and how they have influenced the progress made in the field in the 50 years since its publication. 


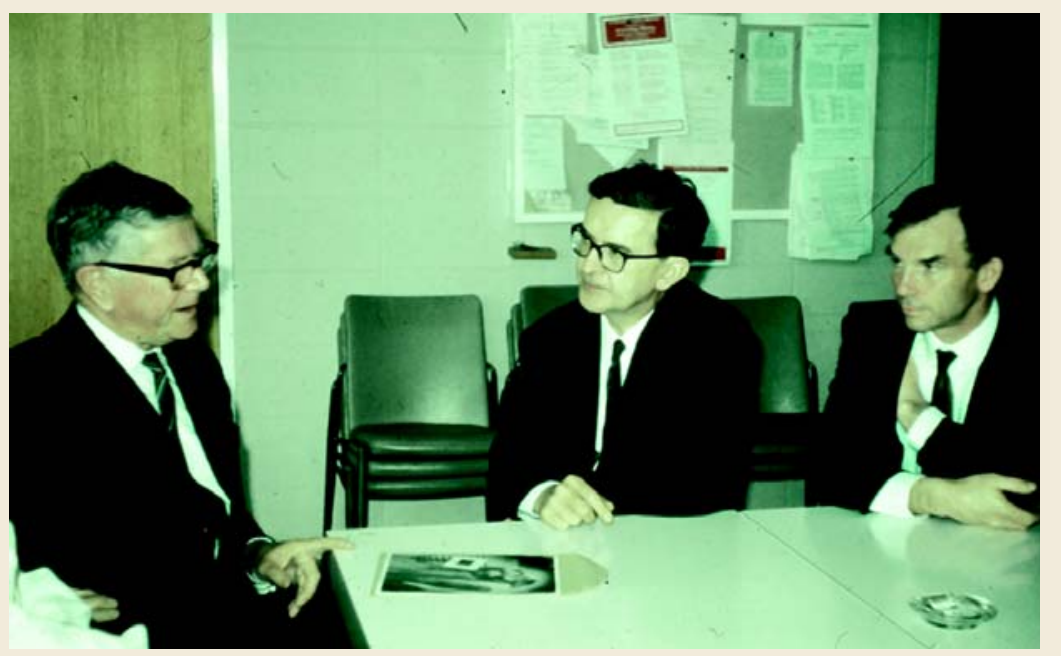

Sir Macfarlane Burnet, Niels Jerne and lan Mackay discussing immunological theories at the Walter and Eliza Hall Institute in the early 1960s

Given the significance of diagnostic testing for autoantibodies that followed the publication of Autoimmune diseases, it is of some interest that this monograph was itself published more than 50 years after Donath and Landsteiner's seminal description of the antibody-mediated autoimmune nature of paroxysmal cold haemoglobinuria. ${ }^{7}$ This observation and perhaps the serological investigations into the nature of the Wassermann and Forssman reactions ${ }^{8}$ were most likely to have contributed to Mackay and Burnet's thinking on the pathogenesis of autoimmunity. Their singular insight was to implicate both the humoral and cellular immune systems in causing tissue damage, as was subsequently so clearly demonstrated through the newly emerging techniques of direct and indirect immunofluorescence.

Many common autoimmune disorders acknowledged today, such as systemic lupus erythematosus (SLE), rheumatoid arthritis and hyperthyroidism, were listed in the monograph. However, there were some notable exceptions, including type I diabetes mellitus, coeliac disease, autoimmune skin disorders (pemphigus, alopecia and vitiligo), antiphospholipid syndrome and the antineutrophil cytoplasmic antibody (ANCA)-associated vasculitides (Wegener's granulomatosis and pauciimmune glomerulonephritis). Several diseases (eg, ankylosing spondylitis, inflammatory bowel disease and psoriasis) were listed as possibly immune — issues that can be considered unresolved today.

Mackay wrote prophetically (page 104):

The fact that SLE encompasses the entire spectrum of immunopathological lesions attributable to forbidden clones makes this a key disease, the further understanding of which could clarify the entire field of autoimmunity.

Fifty years later, based on exhaustive experimental evidence from murine models of lupus, together with improved techniques for autoantibody recognition, our understanding of the genetic, environmental and stochastic factors operative in human lupus (and other autoimmune disorders) is indeed much enhanced.

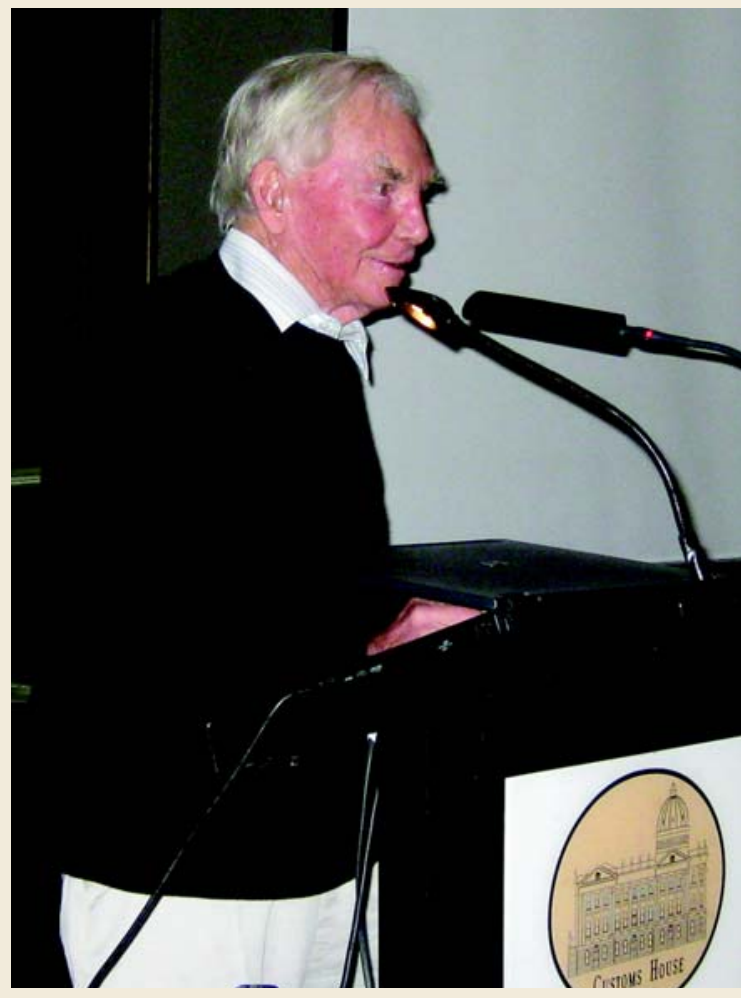

Professor lan Mackay speaking at the 14th Australasian Autoimmunity Workshop in Brisbane, May 2011

Mackay also described what is now known as apoptosis, long before it was formally described by the Australian John Kerr and his colleagues in $1972,{ }^{9}$ as well as the important role of apoptosis in the pathogenesis of SLE (page 100):

A major clue to the pathogenesis of SLE is almost certainly to be found in the lymph nodes, which are enlarged and show focal necrosis and fragmentation of nuclei. The forbidden clone hypothesis would explain the situation as follows. Lymph nodes normally contain damaged pyknotic nuclei undergoing degradation; with the postulated breakdown of the homeostatic mechanism, immunologically competent lymphoid cells carrying forbidden antinuclear patterns are attracted to settle and proliferate in lymph nodes where corresponding nuclear material is being released - in an otherwise healthy individual this release of nuclear material may occur virtually nowhere else than in the lymphoid tissues.

Mackay went on to write (page 101):

Subsequently, as the disease becomes established, forbidden immunologically competent cells and the corresponding circulating antibodies will increase in both number and diversity, since large numbers of different antigenic determinants will be liberated from various types of damaged cells.

This appears to be early recognition of antigenic intermolecular spreading well before the concept was described. ${ }^{10}$ 
Burnet wrote, in relationship to the development of a forbidden clone (page 39):

In health, immunologically competent cells carrying self-reactive patterns are deleted by homeostatic mechanisms. Failure of deletion of such cells, possibly associated with weakness of homeostasis, allows the development of a self-reactive or forbidden clone of cells, potentially capable of causing autoimmune disease. The origin of the forbidden clone is indicated as being due to mutation but it should be emphasized that more precise knowledge in the future may show that the forbidden pattern represents a persistence of one of the original embryonic cell reactivities or the release of a repression rather than a mutation.

Over subsequent decades, the existence of forbidden clones has become problematic, with research concentrating on disturbed homeostasis/ immunoregulation (ie, a "release of a repression" in the Burnet parlance). Hence, in 1983, Sir Gustav Nossal (Burnet's successor at WEHI) concluded: ${ }^{11}$

The complex process of self-tolerance is probably an amalgam of repertoire purging, suppression, sequestration of self antigens, and blockade of receptors by monovalent, non-immunogenic self molecules and other regulatory loops, possibly including anti-idiotype networks. Evolutionary elimination of $\mathrm{V}$ genes with self-reactive potential is also conceivable...

Later that year, even Mackay admitted that the emergence of a forbidden clone arising from somatic mutations in the repertoire of antibody-producing cells is "unlikely as a general explanation for autoimmunity". ${ }^{12}$ However, a recent publication provides likely evidence of a clonotypic origin for a specific anti-Ro epitope in Sjögren's syndrome/SLE. ${ }^{13}$ Perhaps Mackay and Burnet got it right after all.

Mackay and Burnet's 1962 monograph is seen as a founding text and marked the beginning of autoimmunity as a clinical science. Its insightful concepts and novel ideas inspired a generation of clinical and basic immunological researchers. We salute Ian Mackay and Sir Macfarlane Burnet on the 50th anniversary of this seminal publication.

Competing interests: No relevant disclosures.

1 Jacobson DL, Gange SJ, Rose NR, Graham NMH. Epidemiology and estimated population burden of selected autoimmune diseases in the United States. Clin Immunol Immunopathol 1997; 84: 223-243.

2 Rose NR, Mackay IR. Prospectus: the road to autoimmune disease. In: Rose NR, Mackay IR, editors. The autoimmune diseases. 4th ed. New York: Elsevier Academic Press, 2006: xix - xxv.

3 Silverstein AM. Autoimmunity: a history of the early struggle for recognition. In: Rose NR, Mackay IR, editors. The autoimmune diseases. 4th ed. New York: Elsevier Academic Press, 2006: 3-9.

4 Mackay IR, Burnet FM. Autoimmune diseases: pathogenesis, chemistry and therapy. Springfield, Ill: Charles C Thomas, 1963.

5 Burnet FM. The clonal selection theory of acquired immunity. Cambridge: Cambridge University Press, 1959.

6 King LS. Autoimmune diseases; pathogenesis, chemistry and therapy [book review]. JAMA 1963; 184: 81.

7 Donath J, Landsteiner K. [Über paroxysmale Hämoglobinurie] [German]. Münch Med Wochenschr 1904; 51: 1590-1593.

8 Landsteiner K. The specificity of serological reactions. Springfield, III: Charles C Thomas, 1936.

9 Kerr JF, Wyllie AH, Currie AR. Apoptosis: a basic biological phenomenon with wide-ranging implications in tissue kinetics. Br J Cancer 1972; 26: 239-257.

10 McCluskey J, Farris AD, Keech CL, et al. Determinant spreading: lessons from animal models and human disease. Immunol Rev 1998; 164: 209-229.

11 Nossal GJV. Cellular mechanisms of immunologic tolerance. Ann Rev Immunol 1983; 1: 33-62.

12 Mackay IR. Natural autoantibodies to the fore - forbidden clones to the rear? Immunol Today 1983; 4: 340-342.

13 Lindop R, Arentz G, Chataway TK, et al. Molecular signature of a public clonotypic autoantibody in primary Sjögrens syndrome: a "forbidden" clone in systemic autoimmunity. Arthritis Rheum 2011; 63: 3477-3486. doi: 10.1002/ art.30566. 